\title{
Conhecimento de enfermeiros de Unidades de Terapia Intensiva acerca da notificação de eventos adversos
}

\author{
Nurse's knowledge on the notification of adverse events in Intensive Care Unity \\ Giuliano Stellute ${ }^{1}$, Acácia Maria Lima Oliveira Devezas ${ }^{2}$, Luciana Soares Costa Santos ${ }^{2}$, \\ Graziela Ramos Barbosa de Souza², Márcia Mariano de Araújo Kröger ${ }^{3}$
}

\section{Resumo}

Objetivo: Verificar o conhecimento de enfermeiros de Unidade de Terapia Intensiva (UTI) adulto sobre a ocorrência e notificação de eventos adversos (EA). Método: Pesquisa de campo, exploratória, descritiva, de natureza quantitativa, realizada entre treze (13) enfermeiros intensivistas. Os dados foram coletados por meio de um questionário semiestruturado, aplicado em duas UTIs de um Hospital geral e público da cidade de São Paulo, após aprovação pelo Comitê de Ética em Pesquisa da instituição (sob número 797188). Resultados: Conceitualmente, os enfermeiros descreveram EA como erros, não conformidades ou eventos inesperados, causados ao paciente pela equipe médica ou de Enfermagem, direta ou indiretamente, cujas complicações oferecem algum tipo de risco ou dano. A ocorrência de dano real ou potencial à vida do paciente foi percebida por todos os enfermeiros. Especificamente nas UTIs pesquisadas, todos citaram que os EA ocorrem com frequência e que realizam a notificação dos mesmos. Quanto ao tipo, o EA mais comum é a perda de dispositivos terapêuticos. Para oferecer uma assistência de Enfermagem segura ao paciente é essencial que o enfermeiro que atua em UTI amplie seu repertório de conhecimentos técnico-científicos relacionados aos EA

1. Enfermeiro Especialista em Enfermagem na Assistência ao Adulto em Unidade de Terapia Intensiva pela Faculdade de Ciências Médicas da Santa Casa de São Paulo

2. Professor Instrutor da Faculdade de Ciências Médicas da Santa Casa de São Paulo - Graduação em Enfermagem e Pós-Graduação em Enfermagem na Assistência ao Adulto em Unidade de Terapia Intensiva

3. Professor Instrutor da Faculdade de Ciências Médicas da Santa Casa de São Paulo - Pós-Graduação em Enfermagem na Assistência ao Adulto em Unidade de Terapia Intensiva (Prática Clínica)

Trabalho realizado: Faculdade de Ciências Médicas da Santa Casa de São Paulo - Curso de Pós-Graduação em Enfermagem na Assistência ao Adulto em Unidade de Terapia Intensiva

Endereço para correspondência: Giuliano Stellute. Rua Descalvado, $n^{\circ} 73$ - Vila Lucinda - 09241-210 - Santo André - SP - Brasil.E-mail:giuliano.stellute.gs@gmail.com Conflitos de interesse: Declaramos não haver qualquer conflito de interesses. e torne-se capaz de identificar tanto os fatores de risco predisponentes ou existentes no ambiente de cuidado quanto valorize a notificação dos mesmos, para rastrear causas e efeitos, planejar e implementar sistemas para prevenir, corrigir ou mitigar acidentes, lesões ou outras ocorrências que envolvam pacientes, familiares, profissionais e a estrutura assistencial. Também, é fundamental estabelecer não uma cultura punitiva frente à ocorrência de EA, mas uma cultura que estimule a reflexão e aprendizado a partir destes eventos.

Descritores: Segurança do Paciente, Unidades de Terapia Intensiva, Educação em Enfermagem, Gestão da Segurança
Abstract
Objective: This study verified nurses' knowledge in adult Intensive Care Unit (ICU) about the occurrence and repor- ting of adverse events. Methods: Qualitative exploratory field research among thirteen ICU nurses. The data are collected through a semi structured questionnaire, applied in two ICU of a general and public Hospital in the city of São Paulo, after approval by the Research Ethics Com- mittee of the institution (under protocol number 797188). Results: Conceptually, nurses have described adverse events as an error, nonconformities or unexpected events caused directly or indirectly by the medical or nursing staff to the patient whose complications offer some type of risk or harm. The occurrence of factual or potential damage to the patient's life was perceived by all nurses. Specifically, in the ICU surveyed, all mentioned that adverse events occur frequently, and they report the same. As for the type, the most common adverse event is the loss of therapeutic devices. Discussion: In order to offer a safe nursing care, it is essential that nurses working in the ICU expand their repertoire of technical and scientific knowledge related to adverse events and become able to identify both existing and predisposing risk factors in the care setting as well as valorize their notification for causes and effects to plan and implement systems that prevent, correct or mitigate accidents, injuries or other occurrences involving patients, family members, professionals and the care structure. It is also essential to establish a non-punitive culture against 
the occurrence of adverse events, as well as a culture that stimulates reflection and learning from these events.

Keywords: Patient Safety; Intensive Care Units; Education, Nursing; Safety Management

\section{Introdução}

O ambiente da Unidade de Terapia Intensiva (UTI), em função de suas características e estrutura físico-funcional peculiares, está organizado para prover suporte intensivo e vigilância contínua no diagnóstico e tratamento de pacientes criticamente enfermos, ou seja, aqueles que apresentam grave disfunção ou descompensação em um ou mais sistemas orgânicos, independentemente de seu prognóstico, requerendo assistência multidisciplinar especializada, compatível com a maior complexidade assistencial ${ }^{(1-2)}$.

Sendo assim, é essencial à enfermeiro e equipe de Enfermagem da UTI alcançarem um grau de capacitação e qualificação que atendam aos padrões de excelência, qualidade e segurança no cuidado de Enfermagem, capazes de atender às múltiplas demandas relacionadas ao próprio paciente como a seus familiares, à equipe multidisciplinar e à estrutura assistencial necessária.

Além de realizar a gestão dos recursos e do ambiente assistenciais, cabe ainda ao enfermeiro intensivista interpretar a condição clínica dos pacientes sob sua responsabilidade com base em conhecimentos e competências específicos e estabelecer a interface entre a tecnologia disponível e a humanização do cuidado, sendo capaz de tomar decisões assertivas, tanto individualmente quanto em equipe, e desenvolver um trabalho conjunto e coordenado no contexto multidisciplinar focado na minimização de riscos, incidentes e erros.

Desde a publicação do relatório To err is human: building a safer health care system (Errar é humano: construindo um sistema de saúde seguro) pelo Instituto de Medicina dos Estados Unidos (Institute of Medicine - IOM) $)^{(3)}$ no final da década de 90 , que discute a segurança do cuidado de saúde em instituições norte-americanas, muitos países despertaram para o fato de que erros eram frequentes e provocavam milhares de mortes, danos e sequelas irreversíveis, sendo uma importante causa de morte. Isto levou a mudanças nos sistemas de saúde e evidenciou a importância e seriedade do tema segurança do paciente, pelos vultosos números expressos pelos dados epidemiológicos de diferentes países acerca de vítimas de eventos adversos ${ }^{(4-6)}$.

Sendo uma das maiores forças de trabalho em saúde no Brasil e no mundo e responsáveis por expressivo número de ações de cuidado nas instituições de atenção à saúde, os profissionais de Enfermagem precisam estar à frente das discussões sobre esta temática e construir uma prática assistencial preventiva, com identificação precoce de ocorrências e suas complicações e ações corretivas ou mitigantes para esses eventos $^{(6-8)}$

Apesar da velocidade com que publicações sobre segurança do paciente avolumam-se e tornam-se disponíveis a profissionais e instituições, ainda são perceptíveis inconsistências no uso da terminologia padronizada, o que compromete a compreensão de informações sobre o tema ${ }^{(8)}$.

A Classificação Internacional de Segurança do Paciente (International Classification for Patient Safety - ICPS), desenvolvida por um grupo de trabalho da Aliança Mundial para a Segurança do Paciente, da Organização Mundial de Saúde (OMS), apresentou definições para os principais conceitos em segurança ${ }^{(8)}$.

Conforme a ICPS, erro é definido como uma falha em executar um plano de ação ou aplicação de um plano incorreto, que pode ocorrer por se fazer a coisa errada (erro de ação) ou por falhar em fazer a coisa certa (erro de omissão) na fase de planejamento ou na fase de execução. Erros são não intencionais por definição, enquanto violações são atos intencionais (raramente maliciosos) podendo tornar-se rotineiras e automáticas em certos contextos (por exemplo, a não adesão à higienização das mãos por profissionais de saúde) $)^{(8)}$.

Já incidente é um evento ou circunstância que poderia ter resultado ou resultou em dano desnecessário ao paciente. Os incidentes classificam-se como: near miss (incidente que não atingiu o paciente), incidente sem dano (evento que atingiu o paciente, mas não causou danos discerníveis) e incidente com dano ou evento adverso (incidente que resulta em dano ao paciente) $)^{(8)}$.

A quase falha (near miss) e o incidente sem dano decorrem do cuidado à saúde, mas não acarretam lesões mensuráveis ou interferem no tempo de internação. Já o evento adverso (EA) não está relacionado à evolução natural da doença de base e ocasiona lesões mensuráveis no paciente afetado, prolongamento do tempo de internação e, em alguns casos, óbito ${ }^{(9)}$.

Recomendações da Agência Nacional de Vigilância Sanitária (ANVISA) considerando a gravidade e complexidade dos pacientes na Terapia Intensiva, devido o maior volume de procedimentos e intervenções invasivos, a carga e ritmo de trabalho, o uso de tecnologias diagnósticas e terapêuticas, cada vez mais complexas e a própria falibilidade humana, é fundamental estabelecer um sistema para prevenção de erros e incidentes relacionados ao cuidado à saúde, garantindo assim, uma assistência segura e de qualidade ${ }^{(10,11)}$. 
O enfermeiro deve planejar estratégias para identificar, prevenir e gerenciar riscos e programar ações de educação permanente da equipe para reduzir a um mínimo aceitável o risco de dano desnecessário ao paciente, a ocorrência de eventos adversos e as vulnerabilidades do ambiente de cuidado, bem como operacionalizar processos e ferramentas para registro e notificação, promovendo qualidade e segurança do paciente no sistema de saúde

Este estudo teve por objetivo verificar o conhecimento de enfermeiros de Terapia Intensiva sobre eventos adversos, contribuindo para que os profissionais que atuam na assistência direta e na gestão do cuidado direcionem suas competências profissionais para alcançar metas de melhoria contínua da qualidade, bem como beneficiar pacientes, familiares e instituições hospitalares.

\section{Objetivo}

Verificar o conhecimento de enfermeiros de Unidades de Terapia Intensiva Adulto sobre eventos adversos e sobre seu registro e notificação.

\section{Método}

Trata-se de uma pesquisa de campo, exploratória, descritiva, de natureza quantitativa, conduzida entre enfermeiros de duas Unidades de Terapia Intensiva (UTI) Adulto de um hospital geral de grande porte no Estado de São Paulo, que atende exclusivamente pacientes do Sistema Único de Saúde (SUS) de distritos de saúde localizados em seu entorno.

A amostra foi constituída por treze (13) enfermeiros dos três turnos, presentes no período de coleta de dados, convidados e que aceitaram participar da pesquisa pela assinatura do Termo de Consentimento Livre e Esclarecido (TCLE).

A coleta foi realizada em outubro de 2014, após aprovação pela Comissão Científica de Enfermagem da Faculdade de Ciências Médicas da Santa Casa de São Paulo (FCMSCSP) e pelo Comitê de Ética em Pesquisa (CEP) da instituição, sob número 797.188.

Os dados foram coletados por meio de um questionário, que foi entregue pessoal e individualmente por um dos pesquisadores aos enfermeiros e devolvido no mesmo dia da entrega, após preenchimento.

O questionário continha questões fechadas com variáveis relacionadas ao perfil dos profissionais (idade, tempo de graduação, tempo de atuação em UTI e na UTI da instituição, maior titulação obtida e participação em eventos sobre EA e notificação) e aos eventos adversos (quanto à ocorrência de danos ao paciente e natureza dos eventos adversos $\mid$ ) e questões abertas acerca do conhecimento específico do profis- sional sobre EA (concepção, frequência de ocorrência, condutas adotadas frente ao EA, processo para registro e notificação de EA e beneficiados pela notificação). Em algumas questões eram admissíveis múltiplas respostas por um mesmo indivíduo, com variação do número de ocorrências possíveis.

Após a coleta, foi criado um banco de dados eletrônico com uso do software Excel, para apoiar a tabulação, análise das variáveis quantitativas (utilizando-se frequências absolutas e médias para resumir as informações) e qualitativas (frequências absolutas das respostas obtidas e análise descritiva das respostas).

Os resultados foram apresentados sob forma de tabelas e figuras.

\section{Resultados}

Em relação ao perfil da amostra, composta no total por dezoito enfermeiros, treze participantes $(72,2 \%)$ tinham idade entre 26 a 53 anos, com idade média de 35,7 anos, graduados em média há oito anos. O tempo de experiência em UTI variou entre menos de um ano até quatorze anos, com média de seis anos e o tempo de atuação nas UTI da instituição teve uma variação entre menos de um ano até nove anos.

Quanto à maior titulação obtida, não havia mestres e/ou doutores em Enfermagem e 85\% (onze) eram especialistas e destes, 44,4 \% tinham especialização em Terapia Intensiva.

No tocante à participação em eventos com foco em segurança do paciente e notificação de EA, verificou-se que $85 \%$ referiram não ter participado de qualquer evento desta natureza.

Sobre a concepção que estes enfermeiros tinham acerca de EA, quatro (21\%) respostas apontaram como sendo erros causados ao paciente pela equipe médica e de Enfermagem; seis (31,6\%), complicações que oferecem algum risco ao paciente; seis $(31,6 \%)$, danos indiretos e diretos ao paciente e três $(15,8 \%)$, não conformidades e eventos inesperados, conforme mostra a Tabela 1.

Quanto à questão sobre se todo EA gera dano à vida do paciente, apenas $4(31 \%)$ responderam que não.

Na questão onde foram apresentadas diversas opções quanto à da natureza dos EA reconhecidos pelos enfermeiros (relacionada a medicamentos, vigilância do paciente, manutenção da integridade cutânea, metas de segurança), das nove opções dadas, seis delas $(66,7 \%)$ foram assinaladas por todos os enfermeiros; dois deles foram assinalados por quase a totalidade dos participantes $(22,2 \%$ ) e um, por $11,1 \%$. Quando foi assinalada a opção 'Outros', nem sempre foi descrita a natureza do evento. A frequência de distribuição das respostas está apresentada na Tabela 2. 


\section{Tabela 1}

\section{Concepção dos enfermeiros}

quanto à eventos adversos.

São Paulo, 2014.

\begin{tabular}{lcc}
\multicolumn{1}{c}{ Conceito } & $N$ & $\%$ \\
$\begin{array}{l}\text { Complicações e riscos ao } \\
\text { paciente }\end{array}$ & 6 & 31,60 \\
$\begin{array}{l}\text { Dano direto ou indireto ao } \\
\text { paciente }\end{array}$ & 6 & 31,60 \\
$\begin{array}{l}\text { Erro médico e de enfermagem } \\
\text { ao paciente }\end{array}$ & 4 & 21,00 \\
$\begin{array}{l}\text { Evento inesperado, não } \\
\text { conformidade }\end{array}$ & 3 & 15,80 \\
Total & $\mathbf{1 9 *}$ & $\mathbf{1 0 0 , 0 0}$
\end{tabular}

*Admissível mais de uma resposta

Entre os EA citados pelos enfermeiros como ocorrendo com frequência nas UTI do estudo, houve predominância da perda de dispositivos evento este relacionado à vigilância do paciente, seguido por lesões por pressão (integridade cutânea) e EA não descritos, conforme apresentado na Figura 1.

Sobre a frequência de ocorrência de EA nas UTI todos responderam que ocorrem com frequência e a conduta que adotam ao presenciá-los em 52,4\% (11) dos casos foi a realização de registro e notificação do evento, seguida por intervenções para evitar danos irreversíveis (19,04\%). Comunicação à equipe médica e Supervisão de Enfermagem e ações educativas da equipe apareceram com a mesma frequência $(14,28 \%)$, como mostra a Figura 2.

Em relação ao processo como é realizada a notificação dos EA, todos os enfermeiros responderam que a notificação dos EA é realizada por meio de formulário
Tabela 2

Frequência dos eventos adversos reconhecidos pelos enfermeiros. São Paulo, 2014.

\begin{tabular}{lcc}
$\begin{array}{l}\text { Natureza de eventos adversos } \\
\text { reconhecidas }\end{array}$ & $n$ & $\%$ \\
$\begin{array}{l}\text { Erros de identificação do } \\
\text { paciente }\end{array}$ & 13 & 11,40 \\
Erros de medicação & 13 & 11,40 \\
Falhas na comunicação & 13 & 11,40 \\
Procedimentos errados & 13 & 11,40 \\
Perdas de dispositivos & 13 & 11,40 \\
(cateteres/sondas/drenos) & & \\
Quedas & 13 & 11,40 \\
Infecções relacionadas à & 12 & 10,52 \\
assistência à saúde (IRAS) & & \\
Úlcera por pressão ${ }^{* 1}$ & 11 & 9,64 \\
Outros & 07 & 6,14 \\
Interação entre Homem e & 06 & 5,30 \\
Tecnologia & & 100,00 \\
Total & $114 *$ & \\
\hline
\end{tabular}

*Admissível assinalar todas as opções.

${ }^{*}$ A terminologia foi alterada para Lesão por Pressão (LPP) em 2016, após a coleta dos dados.

acessado na rede local de computadores da instituição (intranet), que após preenchido, é encaminhado ao Serviço de Gerenciamento de Risco da Instituição.

Quanto a quem seria mais beneficiado com a notificação de EA foram citados o paciente em $46,15 \%$, seguido pela instituição $(23,07 \%)$, o profissional $(19,23 \%)$ e os familiares $(11,55 \%)$. Os dados são mostrados na Figura 3.

Eventos frequentemente vivenciados pelos enfermeiros nestas UTI.
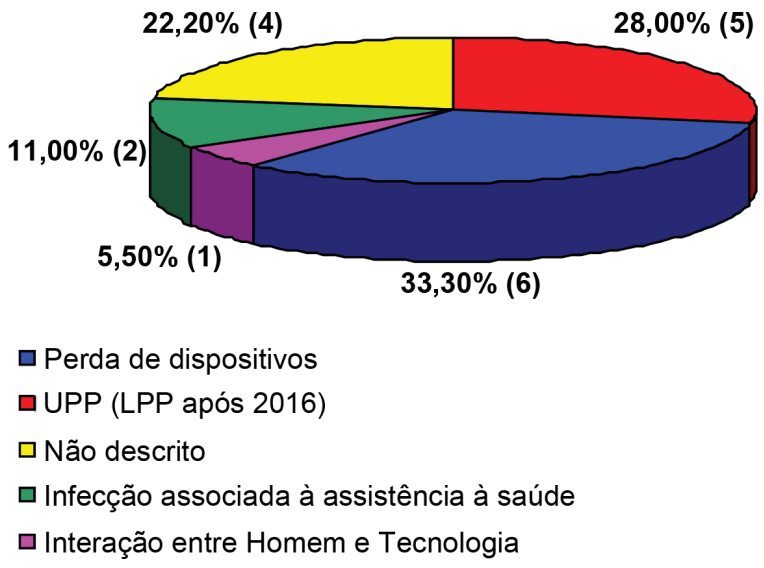

Figura 1 - Frequência de ocorrência de eventos adversos nas UTI. São Paulo, 2014. 


\section{Frequência de condutas adotadas pelos Enfermeiros frente à ocorrência de evento adverso.}

$14,28 \%(3)$

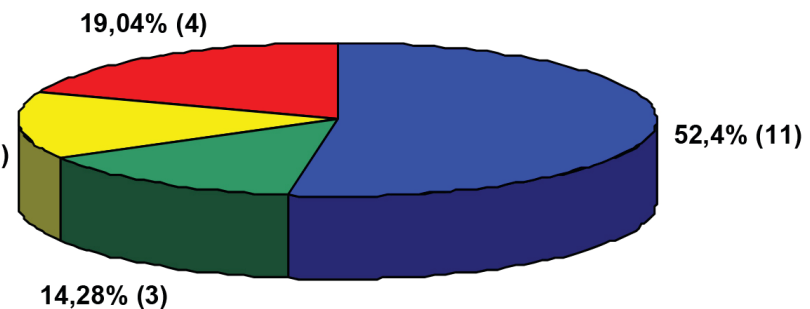

Registro e notificação

Comunica à equipe médica e Supervisão de Enfermagem

$\square$ Orientação da equipe para evitar nova ocorrência

$\square$ Intervenção direta para evitar dano irreversível

Figura 2 - Conduta adotada pelos enfermeiros frente à ocorrência do evento adverso. São Paulo, 2014.

\section{Beneficiados com a notificação dos eventos adversos.}

$\mathrm{N}=\mathbf{2 6}$

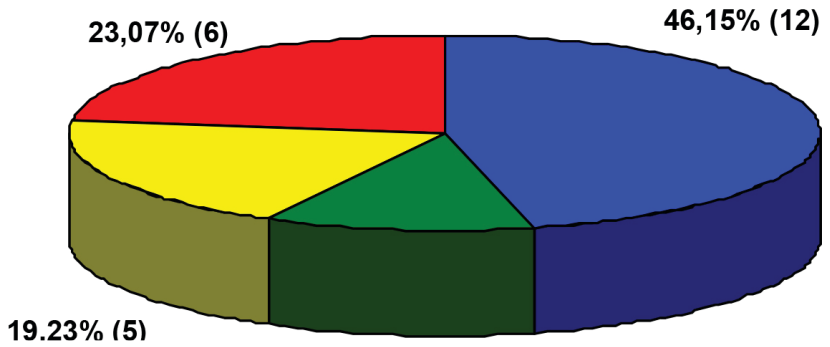

$\square$ Paciente $\quad \square$ Familiares $\quad \square$ Profissional Instituição

Figura 3 - Distribuição dos beneficiados com a notificação dos eventos adversos. São Paulo, 2014.

\section{Discussão}

Em relação ao perfil encontrado entre os enfermeiros da pesquisa, verificou-se que em relação à idade média (35,7 anos, com variação entre 26 e 53 anos), tempo médio de Graduação (oito anos) e de experiência profissional (seis anos) e maior titulação obtida (Especialização), a amostra mostrou-se muito semelhante e consistente ao perfil encontrado na pesquisa nacional Perfil da Enfermagem no Brasil, realizada pelo Conselho Federal de Enfermagem (COFEN) em parceria com a FIOCRUZ em 2013 ${ }^{(12-13)}$ e dados divulgados pelo Conselho Regional de Enfermagem de São Paulo (COREN-SP).

Apesar do tempo médio de Graduação dos enfermeiros ter sido de 8 anos, o tempo médio de atuação em Terapia Intensiva ter sido de seis anos (com variação de menos de um ano até 14 anos) e o tempo de atuação na UTI da instituição ter variado entre menos de um ano até nove anos, um aspecto que deve ser destacado é a inserção de enfermeiros recém-formados na UTI.

O desejável é que, em função da elevada complexidade do paciente e ambiente da UTI, o profissional deva ter uma base sólida de conhecimentos, habilidade em relação aos aspectos tecnológicos e emocionais do cuidado, habilidade para fazer julgamentos clínicos frente a situações que se alteram rapidamente, além de um sistema de valores que o leve a tomar decisões éticas na prática diária ${ }^{(14)}$. O desenvolvimento de com- 
petências clínicas é um processo gradual e, em geral, apenas profissionais com maior experiência clínica estão capacitados à assistência ao paciente crítico, o que torna a inserção de enfermeiros pouco experientes uma prática não recomendável no que diz respeito à segurança do paciente ${ }^{(1,14)}$.

Em relação a este achado, cabe lembrar que não foi investigado se estes enfermeiros haviam atuado na UTI como técnicos em Enfermagem, o que poderia justificar o menor tempo de experiência e atuação na função de enfermeiro.

Um achado positivo é o elevado percentual de especialistas em Terapia Intensiva ou outras áreas nestas UTI, demonstrando uma maior busca por conhecimento para a assistência ao paciente crítico $^{(1-2)}$.

Também, apesar da UTI ser considerada um dos ambientes mais agressivos, tensos e traumatizantes de um Hospital na visão da equipe e de pacientes e familiares ${ }^{(15)}$, os enfermeiros intensivistas da instituição tinham uma variação no tempo de atuação de até 9 anos, o que pode demonstrar que com a vivência, por vezes constante, de situações estressantes, a experiência adquirida amplia a adaptação ao ambiente e permite o desenvolvimento do equilíbrio emocional e resiliência.

A participação dos enfermeiros em eventos relacionados à segurança do paciente e EA indica a necessidade de a instituição investir na educação permanente e capacitação dos enfermeiros nessa temática, para atuar como elemento multiplicador em programas para desenvolvimento da equipe de Enfermagem e multiprofissional e disseminador da cultura de assistência segura ${ }^{(1,6,8)}$.

Quanto à concepção (conhecimento do conceito definido) que os enfermeiros têm acerca eventos adversos mostraram que este conhecimento está presente, mas há inconsistências conceituais como a própria literatura aponta ${ }^{(8-9,16)}$.

Os EA são caracterizados pela presença de dano ao paciente, tanto por não intencionalidade (erros) quanto por intencionalidade (violações), comprometendo a segurança do paciente ${ }^{(8)}$. As respostas dadas pelos enfermeiros mostram alguma forma de concepção acerca do tema, apesar de não apresentarem completa assertividade conceitual à temática de segurança do paciente na assistência à saúde $\mathrm{s}^{(4,6,8,16-18)}$.

Este achado é reforçado pela não percepção da ocorrência de danos ao paciente em decorrência de um EA por doze enfermeiros (67\%) e pela presença de respostas que apontam para iatrogenias (evento associado a alterações patológicas no paciente por um tratamento ou conduta inadequados ao estado e/ou necessidade do paciente) $)^{(16,19-20)}$, ao passo que o evento adverso diz respeito às ocorrências com o paciente em função da ausência da assistência ou de condições estruturais e ambientais ou de uma ação inadequada com dano real ${ }^{(20)}$.

É importante lembrar que uma iatrogenia pode ser um evento adverso, mas nem todo evento adverso é uma iatrogenia ${ }^{(20)}$.

No que se refere à natureza dos $\mathrm{EA}$, conforme os dados da Tabela 2, os enfermeiros foram capazes de reconhecer a maior parte deles, contudo, quer por dificuldade para interpretação dos fatores que ocasionam quer pela existência de lacunas de conhecimento, os EA mais percebidos têm relação a falhas na vigilância do paciente (perda de dispositivos terapêuticos - cateteres, sondas, drenos), danos à sua integridade física (LPP, quedas), falhas em processos assistenciais (erros de identificação ou de medicação, procedimentos errados, IRAS) ou relacionados aos recursos materiais ou estruturais (falhas na comunicação), corroborando dados descritos na literatura ${ }^{(8)}$.

Foram citados ainda, em menor percentual troca de exames laboratoriais, técnica errada na coleta, flebite, reações transfusionais, erro de dispensação de medicamentos, materiais inadequados também referidos na literatura e, muitas vezes, diretamente relacionados aos anteriormente descritos (falhas na identificação do paciente e procedimentos ou medicação errados).

Apesar da literatura apontar que determinados EA (quedas e LPP) podem ser inerentes à condição clínica do paciente crítico (instabilidade hemodinâmica, mobilidade prejudicada, imobilidade prolongada, uso de agentes terapêuticos que comprometem a consciência, percepção sensorial, perfusão, coagulação - drogas vasoativas, sedativas, analgésicas) ${ }^{(16,21-22)}$ e percebido na citação de $28 \%$ dos enfermeiros da pesquisa, nenhum citou EA decorrentes do uso de medicamentos, prática assistencial comum neste tipo de Unidade ${ }^{(19,21)}$.

No que tange à conduta adotada frente à ocorrência de $E A$, pouco mais de um terço respondeu que registra e notifica ( $32,8 \%$ - Figura 2$)$, mas há associação entre condutas (registro e notificação e intervenção para evitar danos ou orientação da equipe ou comunicação à equipe médica e superiores). Observa-se aqui um padrão de ações para gestão do risco, pelo reconhecimento da ocorrência de EA e identificação de padrões e semelhanças entre casos e principais fontes de risco, permitindo delimitar a cadeia de formação do dano ao paciente de forma sistemática e sistematizada $^{(8,10)}$.

O fato de $100 \%$ dos enfermeiros ter respondido notificar os EA, contrasta com o que a literatura aponta em instituições onde existe uma cultura punitiva e os enfermeiros não se sentirem seguros para notificar eventos por existirem punições, apesar de ocorrerem frequentemente EA ou ocorrer indesejável subnotificação e omissão do relato de eventos por medo ou vergonha sentidos pelos profissionais ${ }^{(5,22)}$. A carga de 
trabalho, problemas de ordem institucional e esquecimento também são mencionados como principais fatores para a subnotificação dos $\mathrm{EA}^{(19)}$.

É essencial a superação da cultura punitiva por parte de gestores e implementação de sistemas de registro de EA eficientes, para que as medidas educativas e preventivas necessárias à melhoria da qualidade da assistência e segurança dos pacientes na UTI aconteçam de fato ${ }^{(5)}$, assim como exista um sistema de informação institucional efetivo para gerenciamento de risco e comunicação de EA de forma ágil, permitindo pronta atuação das gerências responsáveis e agilidade na comunicação e acesso à informação ${ }^{(7)}$.

A segurança na assistência é um direito do paciente e, por esta razão, todos são responsáveis e beneficiados com a notificação e registro de EA. Na instituição da pesquisa, quase todos os enfermeiros identificaram primeiramente o paciente como maior beneficiado pela notificação de EA (12 dos 13 participantes, perfazendo o percentual de $46,15 \%$ de respostas, uma vez que mais de uma resposta era admissível), seguido pela instituição (23,07\%), profissionais (19,23\%) e familiares $(11,55 \%)$, de acordo com a Fig. 3.

É importante envolver e educar pacientes e familiares para identificação de EA, pois podem estar aptos a identificar estas ocorrências antes mesmo que os profissionais percebam, incentivando que comuniquem suas percepções e ajudem a reduzir o tempo entre a ocorrência e as ações necessárias ${ }^{(6)}$.

A notificação e análise de EA contribuem para o planejamento e execução de planos para gerenciamento de riscos e investigação efetiva de ocorrências e educação permanente, com benefícios reais a todos os envolvidos na assistência à saúde.

\section{Conclusão}

Acerca da importância do registro e da notificação dos EA, essa pesquisa permitiu perceber que os enfermeiros de UTI têm conhecimento sobre o tema (ainda que o conhecimento sobre conceitos e fatores relacionados seja genérico e restrito) e reconhecem que contribui para a prática segura da assistência de Enfermagem e cuidados à saúde, gerando uma parceria sinérgica entre todos os envolvidos e interessados na segurança do paciente, por estarem bastante próximos a ele.

\section{Considerações finais}

É imprescindível que o enfermeiro intensivista busque constantemente desenvolver e aprimorar seu corpo de conhecimento técnico-científico e competências sobre eventos adversos.

Além de estar bastante próximo ao cuidado, está apto a identificar e rastrear os fatores de risco, notificar a ocorrência de eventos danosos ao paciente e executar um plano de ação capaz de dar suporte ao alcance da qualidade assistencial e segurança do paciente, com benefícios mútuos.

Apesar de ser um tema relevante, é pouco explorado, requerendo que novos estudos sejam realizados para ampliar o conhecimento sobre o tema.

\section{Referências}

1. Viana RAPP, Torre M , editoras. Enfermagem em terapia intensiva: práticas integrativas. Barueri : Manole; 2017. 1024p.

2. Abrahão ALCL. A Unidade de terapia intensiva. In: Cheregatti $\mathrm{AL}$, Amorim CP, organizadoras. Enfermagem em unidade de terapia intensiva. São Paulo: Martinari; 2010. p.16-39.

3. Kohn LT, Corrigan JM, Donaldson MS (Ed.). To err is human: building a safer health system. Washington (DC): National Academy Press; 2000. 312p.

4. Duarte SCM, Stipp MAC, Silva MM, Oliveira FT. Eventos adversos e segurança na assistência de enfermagem. Rev Bras Enferm. 2015;68(1):144-54.

5. Claro CM , Krocockz DVC, Toffolleto MC, Padilha KG. Eventos adversos em unidade de terapia intensiva: percepção dos enfermeiros sobre a cultura não punitiva. Rev Esc Enferm USP. 2011;45(1):167-72.

6. Pedreira MLG, Chanes DC. Enfermagem para a segurança do paciente. In: Harada MJCS, organizadora. Gestão em enfermagem: ferramenta para prática segura. São Caetano do Sul: Yendis Editora; 2011. p. 285-93.

7. Paiva MCMS, Paiva SAR, Berti HW. Eventos adversos: análise de um instrumento de notificação utilizado no gerenciamento de enfermagem. Rev Esc Enferm USP. 2010; 44(2):287-94.

8. Brasil. Agência Nacional de Vigilância Sanitária. Assistência segura: uma reflexão teórica aplicada à prática. [online]. Brasília (DF): ANVISA; 2013. Disponível em: http:/ / www.saude. pr.gov.br/arquivos/File/OSEGURANCA_DO_PACIENTE/ Modulo_1AssistenciaSegura.pdf. [20 nov 2017]

9. Toffoletto MC, Barbosa RL, Andolhe R, Oliveira EM, Janzantte DA, Padilha KG. Fatores associados aos eventos adversos em uma unidade de terapia intensiva. Rev Bras Enferm. 2016; 69(6):977-83.

10. Brasil. Agência Nacional de Vigilância Sanitária. Gestão de riscos e investigação de eventos adversos relacionados à assistência à saúde. [online]. Brasília (DF): ANVISA; 2017. Disponível em http://portal.anvisa.gov.br/documents/33852/3507912/ Caderno+7+-+Gest $\%$ C3\%A3o+de+Riscos+e+Investiga $\%-$ C3\%A7\%C3\%A3o+de+Eventos+Adversos+Relacionados+\%C3\%A0+Assist $\%$ C3\%AAncia+\%C3\%A0+Sa\%C3\%BAde/ 6fa4fa91-c652-4b8b-b56e-fe466616bd57 [20 nov 2017]

11. Brasil. Conselho Regional de Enfermagem de São Paulo. 10 passos para a segurança do paciente [online]. São Paulo: COREN-SP e REBRAENSP; 2010. Disponível em: http://www.coren-sp. gov.br/sites/default/files/10_passos_seguranca_paciente_0. pdf. [20 nov 2017]

12. Machado HM, Oliveira E, Lemos W, Lacerda WF, Aguiar Filho $\mathrm{W}$, Wermelinger $\mathrm{M}$, et al. Mercado de trabalho da enfermagem: aspectos gerais. Enferm Foco. 2016; 7 (Esp):35-62.

13. Gonçalves LA. Segurança do paciente em Unidade de Terapia Intensiva: carga de trabalho de Enfermagem e a sua relação com a ocorrência de eventos adversos e incidentes. Tese (Doutorado) São Paulo: Escola de Enfermagem da Universidade de São Paulo; 2011.

14. Kitahara PH, Kimura M, Padilha KG. Seguimento do enfermeiro graduado na Escola de Enfermagem da USP: sua inserção 
em unidades de terapia intensiva. Rev Esc Enferm USP. 1999. 33(3):284-93.

15. Fundação Oswaldo Cruz (FIOCRUZ). Escola Nacional de Saúde Pública Sergio Arouca. Núcleo de Estudos e Pesquisas de Recursos Humanos em Saúde, Conselho Federal de Enfermagem (COFEN). Perfil da enfermagem no Brasil. [online]. 2003. Disponível em: https://www.portalcoren-rs.gov.br/docs/ Perfil_Enfermagem_DadosRS.pdf [20 nov 2017]

16. Monte PF, Lima FET, Neves FMO, Studart RMG, Dantas RT. Estresse dos profissionais enfermeiros que atuam na unidade de terapia intensiva. Acta Paul Enferm. 2013; 26(5):421-7.

17. Padilha KG, Kitahara PH, Gonçalves CCS, Sanches ALC. Ocorrências iatrogênicas com medicação em unidade de terapia intensiva: condutas adotadas e sentimentos expressos pelos enfermeiros. Rev Esc Enferm USP. 2002; 36(1):50-7.

18. Beccaria LM, Pereira RAM, Cotrin LM, Lobo SMA, Trajano DHL. Eventos adversos na assistência de Enfermagem em uma unidade de terapia intensiva. Rev Bras Ter Intensiva. 2009; 21(3):276-82.
19. Santos AE, Padilha KG. Eventos adversos com medicação em serviços de emergência: condutas profissionais e sentimentos vivenciados por enfermeiros. Rev Bras Enferm. 2005; 58 (4):42933.

20. Silva LD. Indicadores de qualidade do cuidado de enfermagem na terapia intensiva. Rev. Enferm. UERJ. 2003; 11(1):111-6.

21. Roque KE, Melo ECP. Adaptação dos critérios de avaliação de eventos adversos a medicamentos para uso em um hospital público no Estado do Rio de Janeiro. Rev Bras Epidemiol, 2010;13(4):607-19.

22. Gomes FSL, Bastos MAR, Matozinhos FP, Temponi HR, Valásquez-Meléndez G. Avaliação de risco para úlcera por pressão em pacientes críticos. Rev Esc Enferm USP. 2011; 45(2):313-8.

Trabalho recebido: $01 / 02 / 2018$

Trabalho aprovado: 19/06/2018 